\title{
Secondary Electron Imaging in the Helium Ion Microscope
}

\author{
Brad Matola $^{1}$ and David C Joy ${ }^{2}$ \\ ${ }^{1}$ Denison University, Granville, OH 43023 \\ ${ }^{2}$ University of Tennessee, Knoxville, TN 37933
}

Secondary electrons (SE) have been for many years the most user friendly, versatile, and convenient modes of imaging in the scanning electron microscope. The Helium Ion Microscope (HIM) also generates secondary electrons (iSE) and potentially offers significant advantages compared to the conventional SEM including much enhanced spatial resolution and improved image contrast. There are, however, both similarities and important differences between the properties of SE and iSE signals which must be taken into account to optimize imaging.

SE and iSE emissions both occupy the same portion of the energy spectrum from $1 \mathrm{eV}$ up to $50 \mathrm{eV}$, but there are significant differences between the two cases. The SE signal extends up the energy spectrum up to $50 \mathrm{eV}$, but much of this signal is contributed by backscattered electrons. For the iSE signal however the signal spectrum only extends up to 15 to $20 \mathrm{eV}$ because ion backscattering is weak, or absent, at typical beam energies. The yield of secondary electrons is determined by the velocity of the incident particles rather than by their incident energy. Because the mass of a $\mathrm{He}+$ ion is some $750 \mathrm{x}$ greater than that of an electron then, for identical energies, the $\mathrm{He}+$ ions will be travelling at a velocity several orders of magnitude slower than the electrons. The variation of SE and iSE yields with energy move in different directions because they fall on the opposite sides of the secondary electron yield curve. Raising the electron energy $E$ from $1 \mathrm{kV}$ up to $50 \mathrm{kV}$ enhances the beam range by a factor proportional to $\mathrm{E}^{1.6}$, but leads to a fall in the SE yield of more than an order of magnitude. In the ion case, raising the ion energy $\mathrm{E}$ only increases the beam range by a factor proportional to $\mathrm{E}^{0.68}$, but the iSE yield is increased by an order of magnitude.

The Zeiss "ORION NF" Helium Ion Microscope can generate $\mathrm{He}+$ ion beams with energies falling in the range from about $10 \mathrm{kV}$ up to $35 \mathrm{kV}$, while producing an incident beam current in the range $10^{-14}$ to more than $10^{-12}$ amps at the specimen. To capture these signals the ORION employs a conventional Everhart-Thornley (ET) detector which then feeding directly into an Analog to Digital (A/D) converter. A measure of the performance of a signal detector system is the 'Detector Quantum Efficiency' (DQE) which compares the magnitude of the SE signal captured by the detector to the value predicted given the known values of the incident beam current, the beam energy, the dwell time and SE yield variation with energy. For conventional SEMs measured DQE values typically vary from an acceptable value of about 0.6 down to values as low as 0.05 for detectors with the scintillator that have not been regularly replaced. The HIM gave a measured DQE of 0.11 for a $\mathrm{He}+$ ion beam energy of $25 \mathrm{kV}$ which is acceptable but somewhat lower than is ideal. Because the ion beam generates more secondary electron emission than that produced by a comparable electron beam, and because high ion currents can prematurely degrade both the scintillator and the sample, typical incident ion currents on the HIM are chosen to be in the range $10^{-13}$ to $10^{-11}$ amps.

The iSE signals collected by the Everhart-Thornley detector in the HIM are immediately digitized for subsequent manipulation, display and storage. However, as pointed out by Notte (personal communication), because the detector is set to digitize the incoming signal at a fixed time interval of 
100 nanoseconds then, because the incident ion beam current is small, there is a definite possibility that the analog to digital converter will miss some fraction of the arriving signal pulses so degrading the signal to noise ratio of the image. To overcome this problem the incoming electron counts from the detector are simultaneously processed by two separate and distinct signal chains. The first of these performs 'true averaging' of the data by summing the $\mathrm{N}$ successive $\mathrm{A} / \mathrm{D}$ signal pulses which arrive within the chosen sampling time interval, and then divides that sum by $\mathrm{N}$ to give an averaged signal for the time interval. The second channel performs 'true integration' employing the identical signals to those in the averaging channel but operating as a conventional integrator which directly sums the intensity of the $\mathrm{N}$ successive $\mathrm{A} / \mathrm{D}$ conversions signals received within the time period and then outputs that value. An additional operator control labeled 'image intensity' has been added to the usual brightness and contrast controls to control and exploit this detection scheme. Depending on the setting of the image intensity control it is then possible to choose a pure "averaging" signal, or a pure "integration" signal, or to generate a hybrid signal containing both some fraction of both the averaging and the integrating signals. A typical setting of this arrangement sets the averaging signal mode to around $25 \%$ of the signal and hence $75 \%$ to the integrated component and provides a good balance between signal to noise and image detail.

This arrangement offers flexibility for imaging, but having now to control three independent signal components (brightness, contrast, illumination) makes it difficult to perform reliable quantitative studies, because there are some interactions between the three controls and, in the absence of any calibration facility, reliable resetting of the controls to previously used values is difficult [1].

\section{References:}

[1] The authors thank Dr. John Notte of Zeiss, and Dr. Adam Rondinone of the Center for NanoPhase Materials Science at Oak Ridge National Laboratory, for helpful discussions. 